\title{
On-Line Processing of Orthogonal Exercise Electrocardiograms
}

\author{
M. L. Simoons, H. B. K. Boom, and E. Smallenburg \\ Department of Physiology, University of Utrecht, Utrecht, The Netherlands
}

\begin{abstract}
A special program has been developed for on-line processing of orthogonal electrocardiograms during exercise with a small computer system. Special measures were taken to obtain high measurement accuracy and to prevent errors due to baseline drift, skeletal muscle noise or premature beats. The program was tested on exercise electrocardiograms from 47 normal men and 50 patients with coronary artery disease. Single representative complexes were obtained by averaging of beats selected with the aid of the spatial velocity time function of the $Q R S$ complex and the $S T$ segment. The method used is comparable to, but faster than, cross correlation of the spatial velocity time functions. The onset and end of the $Q R S$ complex during exercise were determined using templates derived from the first record of the particular subject. This algorithm resulted in a lower measurement error than other methods for waveform analysis.
\end{abstract}

\section{INTRODUCTION}

Depression of the $S T$ segment of the electrocardiogram (ECG) recorded during exercise in subjects with a normal ECG at rest is considered to be a specific indication for the presence of myocardial ischemia $(1,2,3)$. It also has a prognostic value for the development of ischemic heart disease in otherwise asymptomatic subjects $(4,5)$. However, the interobserver variation in the visual interpretation of exercise ECG's is large (6). This is caused in part by the amount of noise present in the signal and in part by the different criteria employed for classification. With digital computer techniques the signal to noise ratio of the ECG and thus the measurement accuracy can be improved. Also multivariate analysis of ECG measurements can now be performed. It is therefore to be expected that computer assisted interpretation will improve the diagnostic value of exercise ECG's (7).

Averaging of a number of heart beats is often employed to reduce the amount of noise in the ECG caused by skeletal muscle contractions during exercise. However, averaging may cause considerable errors particularly when the time alignment of the beats is not accurate, or when premature beats are present during exercise. Also $Q R S$ complexes may be detected erroneously when sudden baseline shifts take place. Special techniques should therefore be employed to avoid these sources of artifacts.

In this study a new method is presented for the detection of $Q R S$ complexes and for averaging of the exercise ECG. It is based on selection of those $Q R S$ complexes and $S T$ segments which have a similar waveform. In addition an improved method 
for the detection of the onset and end of the $Q R S$ complex is shown to reduce the measurement variability. Both methods are included in a system for on-line analysis of the exercise ECG. The entire system has been designed around a small and economically attractive computer system. At present a PDP $-8 \mathrm{E}^{1}$ computer with $8 \mathrm{~K}$ core memory is used for this purpose in our laboratory (see appendix).

\section{MATERIALS}

The programs described in this study have been tested with rest and exercise electrocardiograms obtained with the Frank lead system in 47 normal men and 50 men with proven coronary artery disease, whose ages ranged from 25 to $65 \mathrm{yr}$. All normal subjects had no history or signs of cardiovascular disease and a normal ECG at rest. All patients had a history of proven myocardial infarction or of severe angina pectoris. Twenty patients were selected because severe narrowing of one or more coronary arteries was shown by a selective cine coronary arteriogram made within two months before or after the stress test. All subjects were exercised on a bicycle ergometer. The workload was increased stepwise $(10 \mathrm{~W} / \mathrm{min}$ or $30 \mathrm{~W} / 3 \mathrm{~min})$ until the maximum tolerable workload for that individual was reached. Several subgroups from this series were used to test different programs.

Group A consisted of 20 subjects who showed premature beats during the test. Eight second samples of the ECG's which included the premature beats were digitized. All possible combinations of pairs of beats in each sample were displayed. By visual inspection it was decided whether the two beats had the same or different waveform of the $Q R S$ complex and the $T$ wave. 1347 pairs of beats classified in this manner were used to test the selective averaging program.

Group B consisted of the averaged electrocardiograms obtained at rest, during exercise at a heartrate of circa 120 beats/min and during maximal exercise from all 97 subjects. These ECG's were used to test the waveform analysis programs. The averaged beats were displayed on an oscilloscope and the $Q R S$ complexes as well as the ends of the $T$ waves were identified by means of a cursor which could be moved over the screen. The ECG's with the indicated points were stored on tape.

Group $C$ consisted of series of 8 to 12 consecutive, unaveraged beats obtained at rest from 13 normal subjects and 13 patients with abnormal ECG's. This series was used to determine the measurement variability after detection of the onset and end of the $Q R S$ complex with different methods.

\section{DETECTION OF $Q R S$ COMPLEXIS}

Three orthogonal leads ${ }^{2}$ were sampled at $2 \mathrm{msec}$ intervals for $20 \mathrm{sec}$. For the detection of the $Q R S$ complexes and the grouping of the beats the leads were filtered with

${ }^{1}$ Digital Equipment Corporation, Maynard, MA.

${ }^{2}$ Frank lead system. 
a recursive digital low pass filter $(8)$. The unfiltered signals were stored on disk. The first zero bandpass of the filter was $50 \mathrm{~Hz}$. The filter required double precision integer (two words of 12 bits each in the PDP-8E computer system) multiplications only.

The absolute values of the differences between consecutive samples in the three leads were computed as well as the product $(S)$ and the sum $(S V)$ of these differences:

$$
\begin{gathered}
S_{t}=\left|X_{t}-X_{t-1}\right| \times\left|Y_{t}-Y_{t-1}\right| \times\left|Z_{t}-Z_{t-1}\right| \\
S V_{t}=\left|X_{t}-X_{t-1}\right|+\left|Y_{t}-Y_{t-1}\right|+\left|Z_{t}-Z_{t-1}\right|
\end{gathered}
$$

where $t$ denotes the sample number.

If $S_{t}$ exceeded a certain threshold during $20 \mathrm{msec}$ the onset of a $Q R S$ complex was detected (Fig. 1). The end of the $Q R S$ complex was detected as the first of 20 consecutive points $(40 \mathrm{~ms})$ after a $Q R S$ onset which were below the threshold. The threshold was set at $10 \%$ of the maximum value of $S_{t}$ in the preceding $Q R S$ complex.
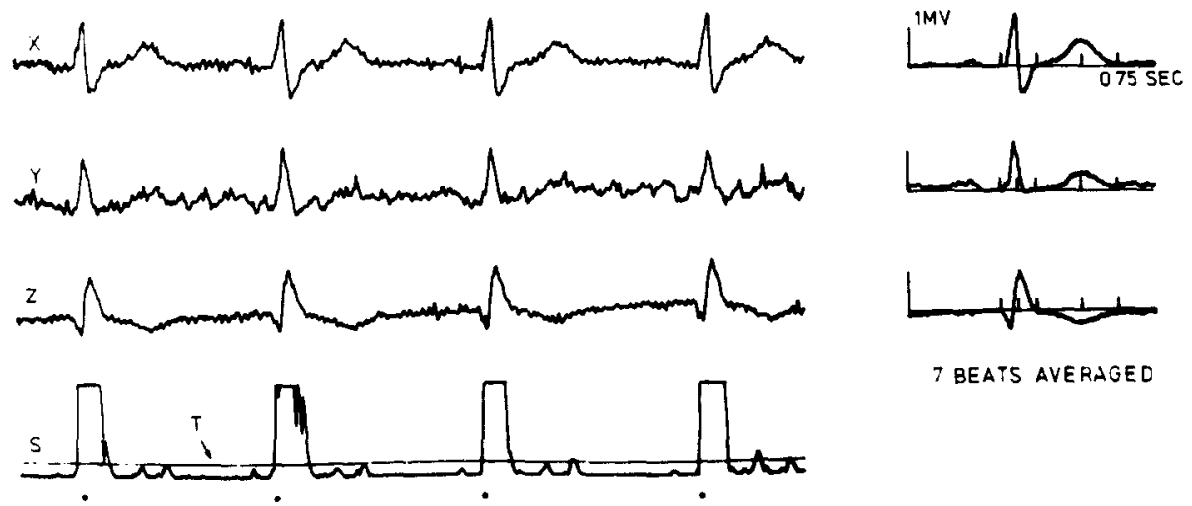

7 BEATS AVERAGED

Fig. 1. Display of the $X, Y$ and $Z$ leads and the product of the filtered difference signals of these leads $(S)$. The detected $Q R S$ complexes are marked with *. The threshold for $Q R S$ detection $(T)$ was set at $10 \%$ of the maximum value of $S$ in the first beat. $S$ is plotted at a relative scale. At the right the result is shown of averaging 7 beats, 4 of which are seen on the left. Note the reduction of noise especially in lead $Y$. The markers in the averaged beat indicate the detected onset and end of the $Q R S$ complex, the maximum spatial magnitude of the $T$ wave and the end of the $T$ wave.

\section{SELECTION OF BeAtS}

The selection of those beats which were averaged in order to obtain a representative beat was performed using the spatial velocity of the heart vector in the $Q R S$ complex and the $S T$ segment. The spatial velocity was approximated by the $S V$ time function given above (9). This saves computer time as no multiplications and square roots need to be performed. The $S V$ curves from 30 samples before up to 97 samples after the onset of each $Q R S$ complex were stored on disk. The maximal value of $S V$ in each beat was normalized to 100 (Fig. 2). The mean value of $S V 30$ to 10 samples 

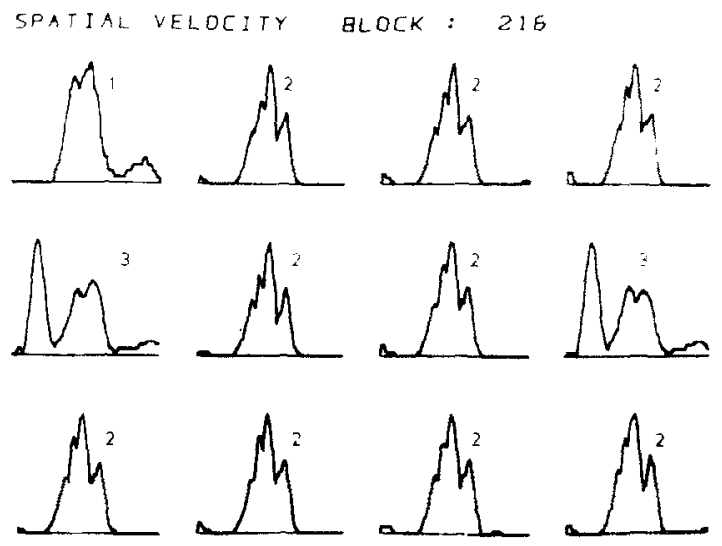

FIG. 2. Display of the filtered spatial velocity curves of 12 consecutive beats. The numbers indicate the groups in which the beats were put by the program. The time scale in $256 \mathrm{msec}$. All maximal amplitudes have been normalised as 100 .

(60-20 ms) before the onset of each $Q R S$ complex was used in order to estimate the noise level for that beat. The beat with the lowest estimated noise level was selected and all other beats were compared with that specific beat. Those with the same waveform of the $S V$ in the $Q R S$ complex and the $S T$ segment were categorized as

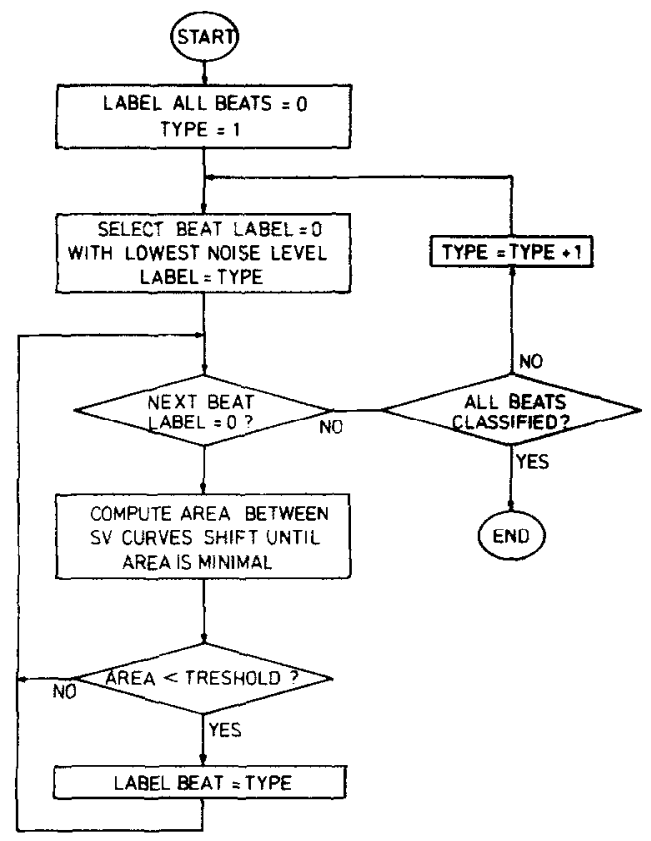

FIG. 3. Flowchart of the program for classification of the detected beats. 
group one. Of the remaining beats the one with the lowest estimated noise level was selected and all beats with a waveform similar to this second selected beat were categorized as group two. This procedure was repeated until all beats were classified (Fig. 3).

In order to compare the waveforms of the $Q R S$ complexes and the $S T$ segments of two beats, the two $S V$ curves were superimposed and the area enclosed by the two

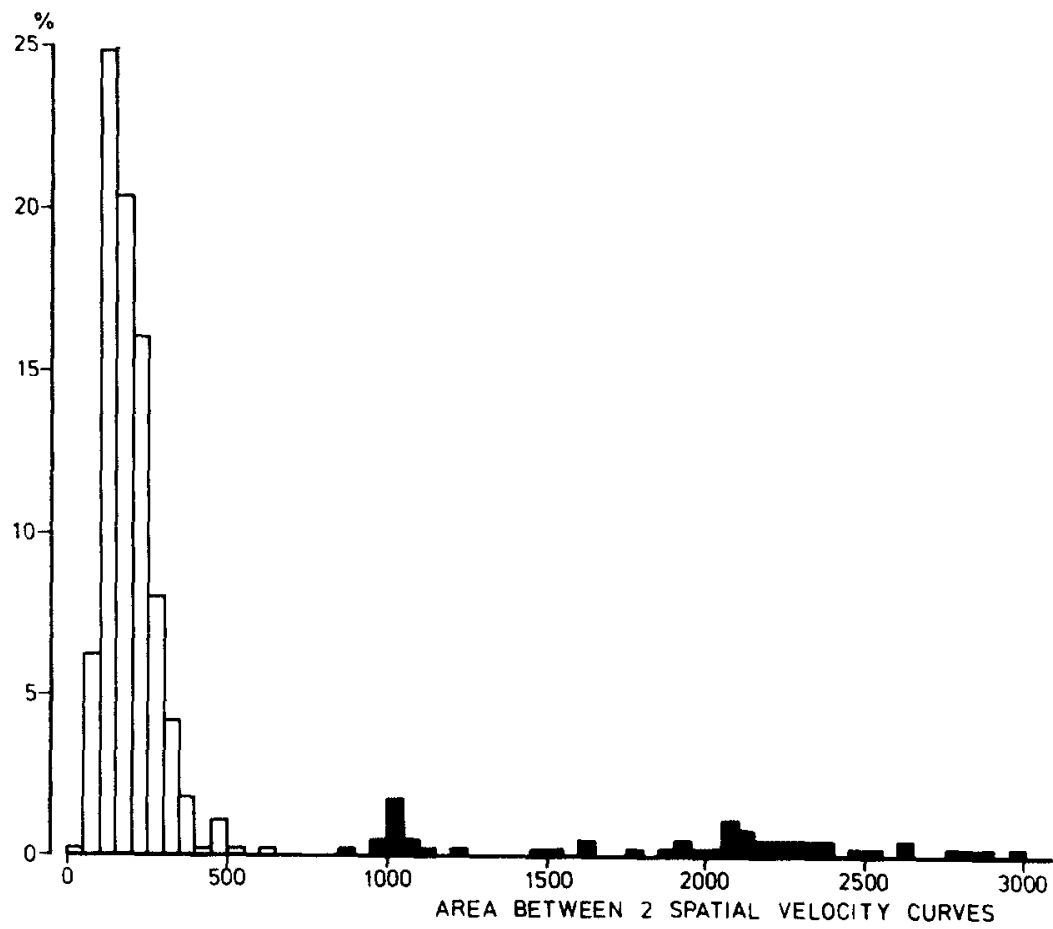

FIG. 4. Histogram of the values of the minimal areas enclosed by the spatial velocity curves of 1347 pairs of beats. The shaded bars indicate pairs of 9 beats with a different configuration.

curves was computed. The relative position in time in which this area was minimal was then searched for. A histogram of this minimal area in 1347 pairs of beats from 20 subjects, test group A, is shown in Fig. 4. A separation between pairs of beats with the same waveform and pairs with different waveforms was obtained when an area of 700 was used as a threshold. This value corresponded to a correlation coefficient between the two $S V$ time functions of 0.94 (Fig. 5).

The positions in which the minimal areas between the $S V$ curves of the 1347 pairs of beats were found, were compared with the positions in which the highest correlation coefficients between the two curves were obtained. In 1193 out of 1347 pairs 
of beats $(89 \%)$ identical positions were found while in 98 pairs $(7 \%)$ a difference of one sample was found. In the remaining 56 pairs $(4 \%)$ a larger difference was obtained. In all these 56 pairs the two beats had different waveforms. The areas between the $S V$ curves of these beats were greater than 1900 and the correlation coefficients smaller than 0.87 .

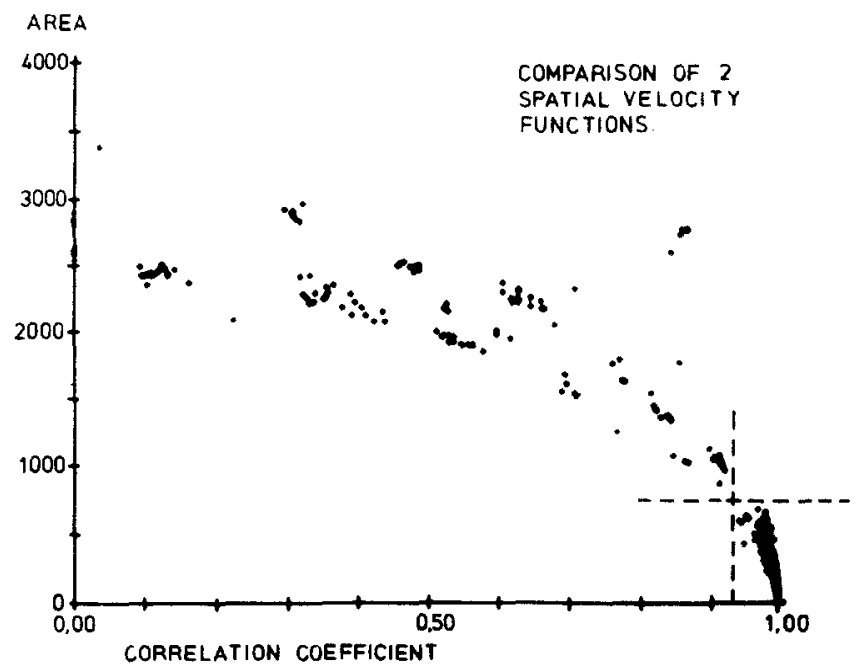

FIG. 5. Plot of the minimal area enclosed by the spatial velocity curves of 1347 pairs of beats $v$ the maximal correlation coefficient. The dotted lines indicate the levels at which pairs of two beats with the same configuration could be separated from those with different configurations. Area $=700$, correlation coefficient $=0.94$.

\section{Averaging of Selected Beats and Quality Control}

The beats which belonged to the largest group were averaged. The relative positions in time in which the smallest areas between the $S V$ curves were obtained, were used as fiducial points. The amount of baseline shift was estimated by computing the level difference between the $P Q$ segments of consecutive beats. If this difference was greater than $0.2 \mathrm{mV}$ in any lead, the beat was rejected. Also all beats were rejected in which overflow of the analog-digital converter due to baseline shift was found. The amount of noise in the averaged beat was estimated by computing the standard deviations of 40 averaged samples in the $S T$ segment.

\section{WAVEFORM ANALYSIS}

The onset and the end of the $Q R S$ complex in the averaged beats were detected from the filtered spatial velocity time functions of these beats. A tentative $Q R S$ 
onset was obtained first by a backward search starting at the time where $S V$ was maximal until 5 consecutive samples were below $7.5 \%$ of the maximal value of $S V$ (Fig. 6). The definitive $Q R S$ onset was then searched for in an interval around the tentative onset. In the first averaged ECG obtained from a given subject the definitive $Q R S$ onset was found by cross correlation with a template for the $S V$ time function (9). This template used in all subjects is further indicated as the "general template."

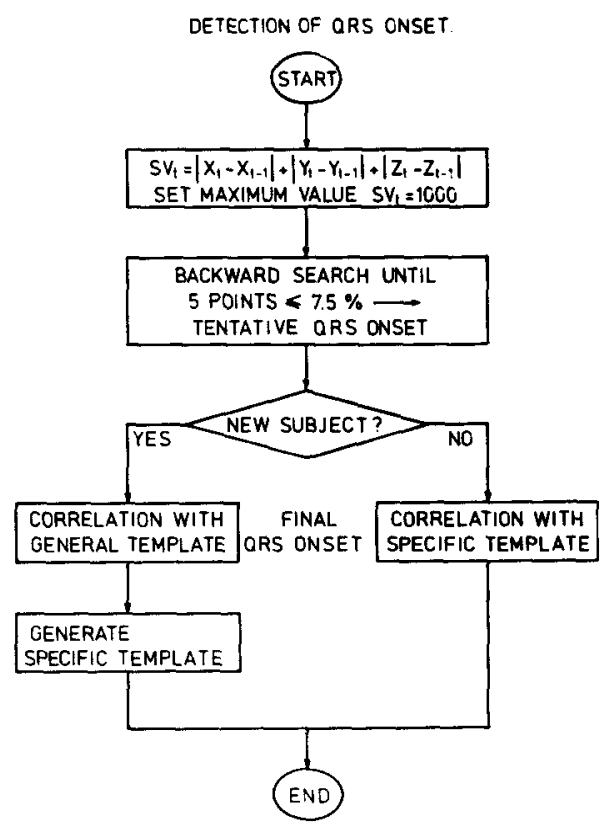

FIG. 6. Flowchart of the programs for detection of the onset of the $Q R S$ complex in the averaged beat. The end of the $Q R S$ complex was detected in a similar way.

The $S V$ function in a window around the onset of the $Q R S$ complex in the first record from a given subject was stored. In the following records of the same subject this signal was used as a "specific template" to detect the $Q R S$ onset. The end of the $Q R S$ complex was detected in a similar way.

This new method for waveform analysis was based on the assumption that the shape of the spatial velocity time function in a given subject does not change during exercise. This was tested in 291 rest and exercise ECG's from 97 subjects (group B). The correlation coefficients between the templates generated from the first record and the spatial velocity time functions in the following records of these subjects were computed. Figure 7 shows that these correlation coefficients were close to 1 when the $S V$ functions around the onset and end of the $Q R S$ complex at rest were compared 
with those during exercise. The shape of the $S V$ around the end of the $T$ wave during exercise, however, was less stable while the $S V$ around the onset of the $P$ wave changed even more. This was caused by fusion of the $P$ and $T$ waves at higher heart rates and by changes of the magnitudes and directions of $P$ and $T$ vectors during exercise. These changes limit the accuracy of the detection of these points in exercise FCG's.
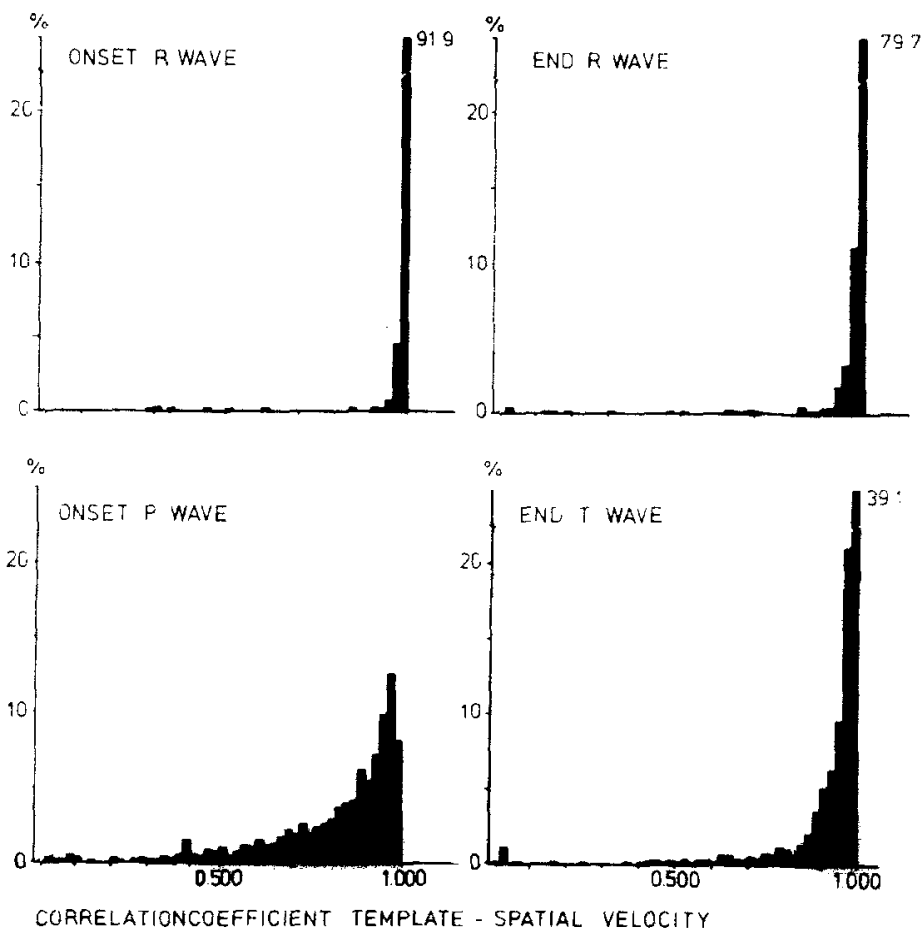

FIG. 7. Histograms of the correlation coefficients between the specific templates generated from the $S V$ at rest and the $S V$ during exercise in 97 subjects. For details see text.

In order to test the value of the use of specific templates for waveform analysis in serial ECG's from one subject, 8 to 12 consecutive beats with similar configurations were analysed in 26 subjects (series $C$ ). In all beats waveform analysis was performed with the general templates and with specific templates obtained from the first beat of each subject (Fig. 8). The mean amplitudes of 8 parts of the $Q R S$ complex with equal durations were computed in all three leads. In Fig. 9 the standard deviations of the 24 time normalised amplitudes from each subject obtained after waveform analysis with the general templates were plotted against the standard deviations obtained after waveform analysis with the specific templates. The latter method resulted in 
lower standard deviations ( $p \leqslant 0.001, F$-test). This shows that a smaller measurement variability was obtained with the specific templates.
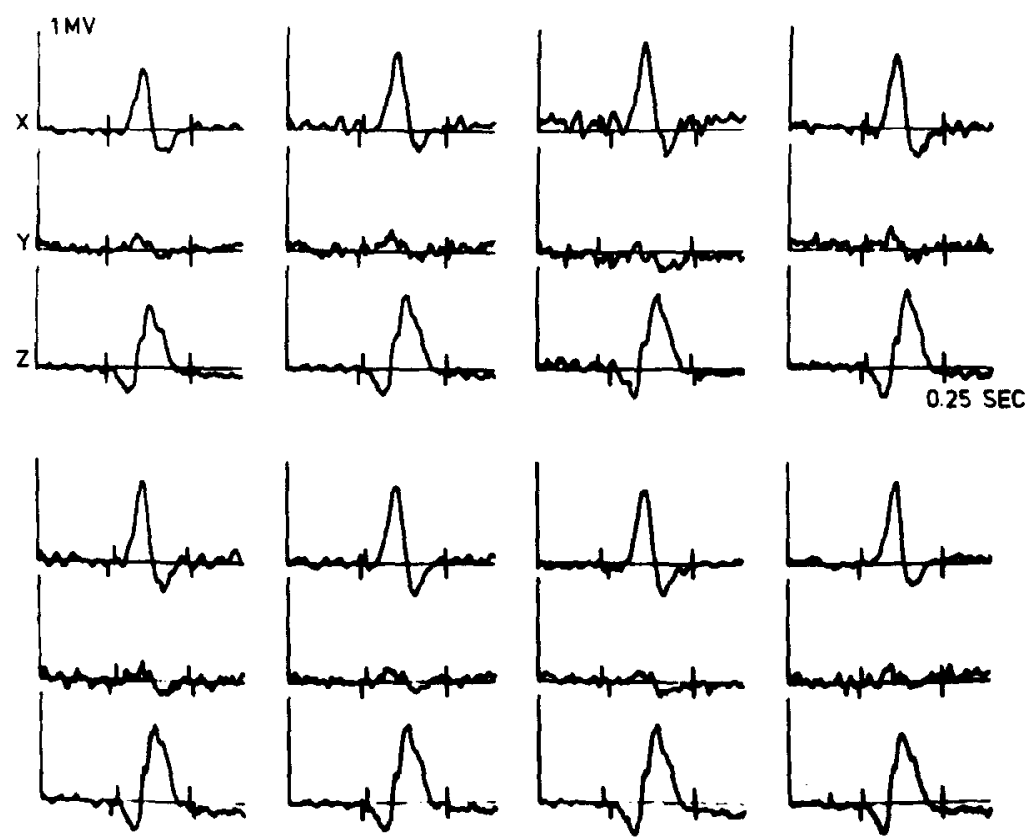

FIG. 8.8 consecutive unaveraged beats from one subject. Amplitude scale: $1 \mathrm{mV}$, time scale: $0.25 \mathrm{sec}$. The markers pointing upward indicate the onset and end of the $Q R S$ complex as detected using general templates only while the markers pointing downward indicate the same points detected using specific templates generated from the $S V$ in the first beat. The positions of the upward markers relative to the $Q R S$ complex vary more than the relative positions of the downward markers. This illustrates the reduction of the variability of the detection of the onset and end of the $Q R S$ complex using the new method for waveform analysis.

\section{DisCussion}

New methods were developed for detection of $Q R S$ complexes, for selective averaging of exercise electrocardiograms and for accurate detection of the onset and the end of the $Q R S$ complex in serial ECG recordings from one subject. The methods were designed for on-line processing of exercise ECG's by means of a small computer system. The analysis of the exercise ECG is performed on-line in order to inform the physician conducting the test immediately when $S T$ segment abnormalities become manifest. Also an on-line system can provide information on the quality of the signals during the test. Measures to reduce baseline drift or noise, usually due to poor electrode connections, can now be taken immediately when these disturbances exceed certain limits. In addition a continuous real time arrythmia detection program 


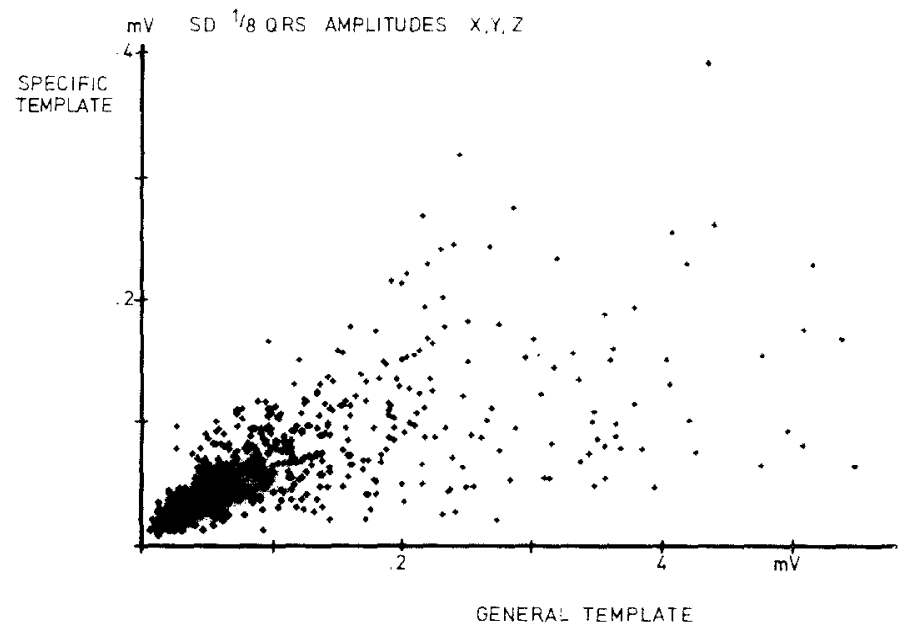

Fig. 9. Plots of the standard deviations of the measurements of 8 time-normalised amplitudes in the $Q R S$ complex, obtained with two waveform analysis methods ( 220 beats from 26 subjects). Horizontal axis : independent waveform analysis of all beats with general templates, vertical axis: waveform analysis with specific templates derived from the first beat of each subject.

which will permit a quantitative analysis of rhythm abnormalities and conduction defects during exercise tests, is under development.

The detection of $Q R S$ complexes was performed in the program with the aid of the product of the difference signals of the three orthogonal ECG leads. This signal exceeds the threshold for $Q R S$ detection when simultaneous changes occur in at least two leads. Analogue $Q R S$ detectors have been developed which use the same principle $(10,11)$. Although these save computer time, software $Q R S$ detection was chosen because it enables the development of a real time arrythmia detection program.

The selection of the beats to be entered into the averaged representative complex is based on the waveform of the spatial velocity time function of the $Q R S$ complex and the $S T$ segment. Brody has described a method in which the detected beats are compared with a normal beat of the same subject by means of a "waveform index" (12). A normal beat is selected by the operator at the start of the test. However, this approach will fail when the $Q R S$ complex changes during exercise, e.g., when a complete or incomplete bundle branche block appears. Methods which are based on clustering of the $Q R S$ complexes $(13,14)$ do not have this disadvantage. In addition these methods, like the one described in this paper, permit averaging and analysis of several types of beats apart from the most frequent type. A disadvantage of the clustering methods is that these use only a few measurements from the $Q R S$ complex (13) and the $S T$ segment (14) while the method described in this study is based on the shape of the whole $Q R S$ complex and the $S T$ segment. If large muscle spikes or 
sudden baseline shifts were present in the $Q R S$ complex or $S T$ segment of a given beat, the $S V$ function of this beat would differ from the $S V$ function of the other beats, and therefore the beat would not be entered into the averaged complex.

The reliability of many measurements from the $Q R S$ complex and the $S T$ segment in the ECG depends on the accuracy of the detection of the onset and end of the $Q R S$ complex. Several methods have been developed for this purpose $(9,13,15)$. We have observed that small changes of the $Q R S$ complex due to noise or respiration sometimes cause a shift of a few milliseconds of the onset or offset of the $Q R S$ complex as detected by two of these methods $(9,13)$. This is illustrated in Fig. 8 . These differences increase the standard deviation of the measurements from the $Q R S$ complex. A reduction of the standard deviation of these measurements could be obtained by waveform analysis in consecutive $Q R S$ complexes using templates which had been derived from the first beat of the series (Fig. 9). The value of this new method for $Q R S$ waveform analysis was illustrated by a study of the change of the duration of the $Q R S$ complex during exercise (16). With the method described by van Bemmel et al. (9) a shortening of the $Q R S$ complex of $2.2 \pm 8.8 \mathrm{msec}$ was found and with the method of Wolf et al. (13) a shortening of $2.4 \pm 8.7 \mathrm{msec}$. These changes were not significant. However, with the new method it was shown that the $Q R S$ complex during submaximal exercise (heart rates $80 \%$ of age predicted maximal heart rate) shortened with $2.7 \pm 2.0 \mathrm{msec}(p \leqslant 0.001)$. So the reduction of the measurement variability obtained with the new method for waveform analysis permitted a better evaluation of the changes of the ECG during exercise. This method may also be of value for the assessment of changes in serial ECG's from one subject over a longer period (17).

\section{APPENDIX}

The programs described in this paper were implemented on a PDP-8E computer system which is presently used for on-line processing of exercise ECG's at our laboratory. The computer system consists of $8 \mathrm{~K}$ core memory, a KE- 8 arithmetic unit, a RF-08 disk, two dectape units, an AX-08 A-D converter, a digital clock, a storage display unit and a teletype. The programs were written in PAL8 assembler language. Some programs were developed using FORTRAN 2. The layout of the programs in core is shown in Fig. 10.

The ECG system is compatible with the OS- 8 operating system. The input-output handlers were core resident as well as the program which handled the overlay's and the SAMPLE program.

The program SETUP initiates a number of variables which are used by the other programs. Some of these are: the number of ECG channels to process (3-6), the number of other channels ( $2-5$ channels for pulse waves, heart sounds and respiratory signals), the sample interval (usually $2 \mathrm{msec}$ ) and the duration of each sample period (usually $20 \mathrm{sec}$ ). The next overlay performs the CALIBRATION of the analog input 
channels. The program ADMINISTRATION stores data like patient code, age, sex and clinical data entered through the keyboard.

Each SAMPLE period is started by a command through the keyboard. A trigger signal to the A-D converter can also be used. During sampling the workload and some other data could be entered. When sampling is finished the $Q R S$ complexes in the signal are detected (DETECTION) and the SELECTION of different types of

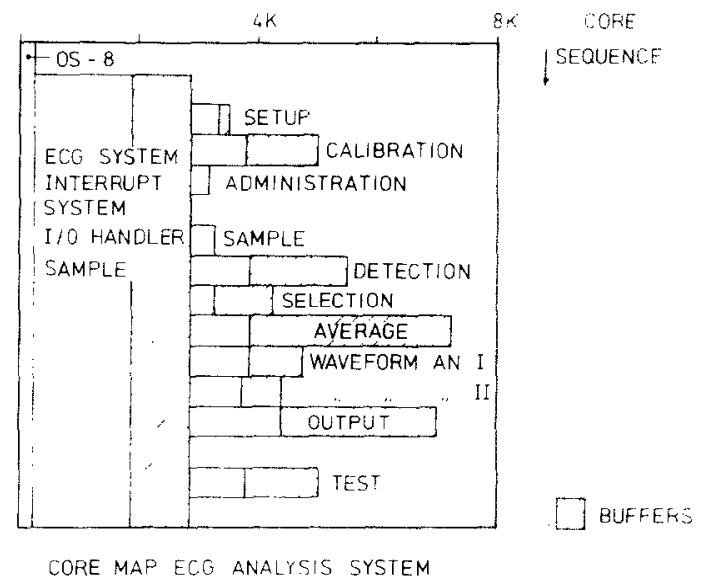

FIG. 10. Layout of the programs for on-line prosessing of exercise ECG's with a PDP-8E computer system. The length of each program in core is indicated horizontally, the sequence of the programs is indicated vertically. NB: The blocks do not represent the actual position of the program in core. Also the actual execution times of the overlay's differ, which is not shown in the figure.

beats is performed. The program AVERAGE averages all beats of the dominant type after rejection of those beats with A-D converter overflow or excessive baseline drift. Other types can also be averaged. The WAVEFORM ANALYSIS is performed on the averaged representative beat.

Finally the averaged beats are stored on dectape and displayed (OUTPUT) after which a new sample can be taken. For quality control purposes the drift and the noise level of the $S T$ segments of the averaged beats were computed. These values are displayed and stored together with the averaged beats. In addition the correlation coefficients obtained in the waveform analysis program are stored. The total processing time for three ECG leads sampled during $20 \mathrm{sec}$ is approximately one minute.

Several programs were available for TEST purposes. One programs stores all sampled data on the second dectape. This was a very useful feature while developing the system.

\section{ACKNOWLEDGMENTS}

The ECG's from the 50 patients used in this study were recorded at the exercise laboratory of the Thoraxcenter of the Erasmus University in Rotterdam. The 47 normal subjects were tested at 
the Occupational Health Service of the habour of Rotterdam. The authors are grateful to Prof. P. G. Hugenholtz and Dr. J. Pool (Thoraxcenter) and Dr. J. Baart (Occupational Health Center) for their cooperation, and for technical and secretarial assistance given by Mrs. M. Muste and Mrs. P. Cornelissen.

Part of the programs were developed by the first author (M. L. Simoons) at the Biophysics and Bioengineering Research Laboratory of the Dalhousie University, Halifax, Nova Scotia, Canada, Director P. M. Rautaharju, M.D., Ph.D.; during a post doctoral fellowship supported by the Dutch Organisation for the Advancement of Pure Research (Z.W.O.).

\section{REFERENCES}

1. Ascoop, C. A., Simoons, M. L., Egmond, W. G., And Bruschke, A. V. G. Exercise test, history and serum lipid levels in patients with chest pain and normal electrocardiogram at rest. Amer. Heart J. 82, 609-617 (1971).

2. Mason, R. E., Likar, I., Biern, R. O., and Ross, R. S. Multiple lead exercise electrocardiography: Experience in 107 normal subjects and 67 patients with angina pectoris, and comparison with coronary cinearteriography in 84 patients. Circulation 36, 517 (1967).

3. Kassebaum, D. G., Sutherland, K. I., And Judkins, M. P. A comparison of hypoxemia and exercise electrocardiography in coronary artery disease: Diagnostic precision of the methods correlated with coronary angiography. Amer. Heart. J. 75, 759 (1968).

4. Blackburn, H. W., Taylor, H. L., and Keys, A. Prognostic significance of the post-excercise electrocardiogram: Risk factors held constant. Amer. J. Cardiol. 25, 85 (1970).

5. Robi, G. P., Marks, H. H., and Mattingly, T. W. The value of the double standard two-step exercise test in the detection of coronary disease: A clinical and statistical follow-up study of military personnel and insurance applicants. Trans. Ass. Life Insur. Med. Dir. Am. 40, 52 (1956).

6. BlackBurn, H. and a technical group, The exercise electrocardiogram: Difference in interpretation. Amer. J. Cardiol. 21, 871 (1968).

7. BLACKBURN, H. Measurement in exercise electrocardiography. The Ernst Simonson Conference, Springfield Il. Charles C. Thomas, 1969. pp. 220-254

8. Lynn, P. A. Recursive digital filters for biological signals. Med. Biol. Eng. 9, 37-43 (1971).

9. Bemmel, J. H. van, Talmon, J. C., Duisterhout, J. S., and Hengeveld, S. J. Template waveform recognition applied to ECG/VCG analysis. Comp. Biomed. Res. 6, 430-441 (1973).

10. Winter, D. A., AND Trenholm, B. G., Reliable triggering for exercise electrocardiograms. IEEE Trans. Biomed. Eng. BME 16, 75-79. (1969).

11. Brandon, C. W., AND Brody, D. A. A hardware trigger for temporal indexing of the electrocardiographic signal. Comp. Biomed. Res. 3, 47-57 (1970).

12. Brody, D. A. The laboratory computer in electrocardiography. In: "Clinical Electrocardiography and Computers," (C. A. Caceres, L. S. Dreifus, Eds.), pp. 401-412. Academic Press, New York, (1970).

13. Wolf, H. K., Macinnis, P. J., Stock, S., Helppi, R. K., and Rautaharju, P. M. Computer analysis of rest and exercise electrocardiograms. Comp. Biomed. Res. 5, 329-346 (1972).

14. Bemmel, J. H. van, ANd Hengeveld, S. J. Clustering algorithm for QRS and ST-Twave form typing. Comp. Biomed. Res. 6, 442-456 (1973).

15. Cornfield, J., Dunn, R. A., Batchlor, C. M. and Pipberger, H. V. Multigroup diagnosis of electrocardiograms. Comp. Biomed. Res. 6, 97-120 (1973).

16. Simoons, M. L., AND RautaharJu, P. M. Changes of P-QRS and QRS-T intervals and QRS duration during exercise and recovery. In preparation.

17. Pryor, T. A., Lindsay, A. E., And England, R. W. Computer analysis of serial electrocardiograms. Comp. Biomed. Res. 5, 709-714 (1972). 